\title{
ANÁLISE ESPACIAL DA FRAGMENTAÇÃO FLORESTAL DA BACIA DO RIO UBÁ - RJ
}

\author{
SPATIAL ANALYSIS OF FOREST FRAGMENTATION OF UBÁ WHATERSHED - RJ STATE
}

\author{
Milton Marques Fernandes ${ }^{1}$ Márcia Rodrigues de Moura Fernandes ${ }^{2}$
}

\begin{abstract}
RESUMO
O objetivo deste estudo foi mapear e analisar uso da terra e a estrutura da paisagem florestal na Bacia Hidrográfica do Rio Ubá - RJ, através de métricas da paisagem. O mapeamento do uso da terra e dos fragmentos florestais foi realizado através de uma imagem CBERS 2B/HRC. Para o cálculo dos índices de ecologia, foi utilizado o programa FRAGSTATS 4.2®. Os fragmentos mapeados foram divididos em classes de tamanho: 1-fragmento muito pequeno, menor que 5 ha; 2 - fragmento pequeno, entre 5 e 10 ha; 3 - fragmento médio, entre 10 e 100 ha e 4 - fragmento grande, maior que 100 ha. As análises quantitativas por meio de métricas da paisagem foram feitas com as métricas de classe: área, forma, área central, agregação e diversidade, sendo a área central obtida em diferentes simulações de efeito de borda $(20,40,60,80,100$ e $140 \mathrm{~m}$ ). Foram mapeados 47 fragmentos florestais em toda a Bacia do Rio Ubá, representando 23,65\% de cobertura florestal. Os fragmentos médios apresentaram maior número (25), seguidos pelos fragmentos de tamanho pequeno (9), muito pequeno (7) e, somente dois fragmentos grandes. A Bacia do Rio Ubá possui um predomínio de uma matriz antrópica representada por pastagem, pequeno percentual de cobertura florestal com fragmentos de tamanho médio entre 10 e 100 ha, com alto grau de fragmentação florestal da paisagem. Os fragmentos médios são a classe mais numerosa e com maior área total, constituindo a única classe de tamanho que mantém uma distância menor que $100 \mathrm{~m}$ entre si. Os fragmentos muito pequenos e pequenos apresentaram formatos mais regulares, porém, com maior densidade de borda. Em relação às métricas de área central, os fragmentos muito pequeno e pequeno perderam totalmente sua área central, estando completamente sob efeito de borda considerando uma distância de borda de $140 \mathrm{~m}$. Uma alternativa no contexto da Bacia do Rio Ubá é a utilização dos fragmentos florestais muito pequenos e pequenos como corredores ecológicos para interligação aos fragmentos médios.
\end{abstract}

Palavras-chave: fragmentos florestais; Mata Atlântica; índices de ecologia da paisagem.

\begin{abstract}
This study aimed at mapping and analyzing the use of the land and the forest landscape structure in Ubá river basin - RJ state, through landscape metrics. The mapping of the land use and of the forest fragments were performed through a CBERS 2B/HRC image. To calculate the ecology indexes, the program FRAGSTATS $4.2 \AA$ was used. The mapped fragments were divided by size classes: 1- very small, smaller than 5 ha, 2 - small fragment, between 5 and 10ha, 3- medium fragment, between 10 and 100 ha, and 4- big fragment, bigger than 100ha. The quantitative analysis through the landscape metrics were performed with the class metrics: area, shape, central area, aggregation, and diversity, obtaining the central area in different simulations of edge effects $(20,40,60,80,100$ and $140 \mathrm{~m}) .47$ forest fragments were mapped through all Uba river basin, representing $23.65 \%$ of forest cover. The medium fragments presented the greatest number (25), followed by the small ones (9), very small ones (7), and only two big ones. Ubá river basin has a predominance of an anthropogenic matrix represented by grasslands, a small percentage of forest cover with medium fragments
\end{abstract}

1 Engenheiro Florestal, Dr., Professor Adjunto do Departamento de Ciências Florestais, Centro de Ciências Agrárias, Universidade Federal de Sergipe, Av. Marechal Rondon, s/n, CEP 49100-000, São Cristovão (SE), Brasil.miltonmf@gmail.com

2 Engenheira Florestal, Doutoranda em Ciências Florestais, Departamento de Ciências Florestais e da Madeira, Centro de Ciências Agrárias e Engenharias, Universidade Federal do Espírito Santo, Av. Governador Carlos Lindemberg, 316, CEP 29550-000, Jerônimo Monteiro (ES), Brasil. marciarmfe@gmail.com 
between 10 and 100 ha, with a high degree of forest landscape fragmentation. The medium fragments are the most numerous class with a wider total area accounting for the only size class that maintains a distance shorter than $100 \mathrm{~m}$ between themselves. Even though the very small fragments and the small ones presented more regular shapes, they had a greater edge density. In relation to the central area metrics, the very small and small fragments totally lost their central area, being completely under the edge effect considering an edge distance of $140 \mathrm{~m}$. An alternative in the context of Ubá river basin is the use of very small and small fragments as ecological corridors for the link with the medium fragments.

Keywords: forest fragments; Atlantic forest; indexes of landscape ecology.

\section{INTRODUÇÃO}

A Mata Atlântica brasileira compreende uma região heterogênea que incluiu uma ampla variedade de fisionomias e composições florestais. No passado constituiu uma das maiores florestas tropicais das Américas, ocupando cerca de 150 milhões de hectares distribuídos em $3.300 \mathrm{~km}$ ao longo da Costa Atlântica Brasileira (METZGER, 2009), restando aproximadamente 11,7\% da vegetação original, que se encontra distribuída, em sua maioria, em pequenos fragmentos florestais $(<50$ ha $)$, o que evidência a urgente necessidade de implementar ações de conservação e restauração para mitigar esta situação (RIBEIRO et al., 2009).

Ao longo do tempo e especialmente nos últimos cinquenta anos, a Mata Atlântica brasileira apresentou grandes taxas de desmatamento e altos níveis de fragmentação por conta do desenvolvimento urbano e expansão agropecuária (SILVA; SANTOS; MORAES, 2014). Após a decadência da cultura do café, a pecuária tornou-se uma atividade importante na região do Vale do Paraíba no Rio de Janeiro, que também apresenta polos industriais. Assim, o histórico de ocupação do Vale do Paraíba gerou a perda de habitat e a fragmentação florestal (DEVIDE et al., 2014). A região possui atualmente uma paisagem que é comumente observada na Mata Atlântica, pois apresenta uma reduzida cobertura florestal, dispersa em meio a pastagens, áreas agrícolas e urbanas (RIBEIRO et al., 2011).

A expansão da fronteira agrícola, com a retirada da vegetação nativa, ou a substituição por outro tipo de uso da terra, tem agravado o processo da fragmentação florestal e provocado consequências negativas nos diferentes compartimentos da natureza, afetando inúmeras espécies da fauna e da flora. A Floresta Atlântica é um dos biomas que tem sofrido grande impacto, mas, mesmo assim, continua sendo responsável por garantir serviços ambientais essenciais à vida humana e à qualidade de vida das pessoas que nele vivem (SOARES et al., 2011).

Isto tem feito com que a paisagem do bioma Mata Atlântica seja comprimida para pequenos fragmentos circundados por diferentes formas de matrizes do entorno. As barreiras ocasionadas pela fragmentação diminuem a dispersão dos organismos entre os remanescentes florestais, resultando em mudanças na estrutura e na função das comunidades florestais (TABARELLI et al., 2010).

O estudo da análise da paisagem sobre fragmentos florestais na área de estudo, pode representar significativo avanço para a compreensão das relações espaciais e a dinâmica florestal destas porções insulares, por causa do desmembramento da Floresta Atlântica (BEZERRA et al., 2011).

A análise dos padrões da fragmentação florestal pode ser feita através das métricas da paisagem, consideradas como base para análises que visam quantificar a estrutura da paisagem, pois fornecem suporte científico para evidenciar a quantidade e qualidade dos fragmentos com base na ecologia da paisagem considerando principalmente, o tamanho do fragmento, a área central, a forma e o grau de isolamento dos fragmentos (SILVA; SOUZA, 2014).

As mudanças das áreas de borda provocam alterações no meio abiótico, como o microclima, incidência de ventos, umidade e radiação solar; e no meio biótico, que são decorrentes das transformações impostas ao meio físico, provocando variações diretas, que correspondem à mudança de distribuição e densidade de indivíduos de uma mesma espécie (ETTO et al., 2013).

Nesse sentido, o uso de métricas e índices de ecologia da paisagem justifica-se para promover um zoneamento no emprego desses recursos para a manutenção da biodiversidade, pois a análise dessas métricas permite auxiliar na definição de técnicas de manejo visando recuperar e/ou conservar os remanescentes florestais (JUVANHOL et al., 2011). 
Diante do exposto, o objetivo desse estudo foi mapear e analisar a estrutura da paisagem florestal na Bacia Hidrográfica do Rio Ubá - RJ, por meio de índices métricos da paisagem.

\section{MATERIAL E MÉTODOS}

\section{Localização e caracterização da área de estudo}

A bacia hidrográfica do Rio Ubá está localizada na região sul do Estado do Rio de Janeiro e é parte integrante da bacia hidrográfica do Rio Paraíba do Sul. Ela possui 8.585 ha e localiza-se nos municípios de

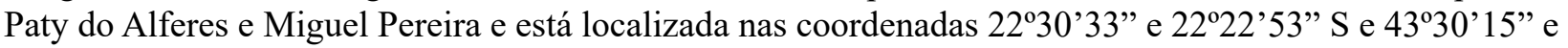
$43^{\circ} 20^{\prime} 50^{\prime}$ "W (Figura 1).

A região está inserida no domínio da Floresta Ombrófila Densa, que revestia toda a área da Serra do Mar, desde o nível do mar até altitudes acima de 1500 metros. Atualmente, a vegetação de floresta reveste os trechos de maior declividade das formações da Serra do Mar. Nas regiões menos acidentadas cedeu lugar a monoculturas, com destaque para plantações de café, cana-de-açúcar e olerícolas. Com o declínio da agricultura, as áreas agrícolas foram substituídas por pastagens e capoeiras, e em determinados locais houve certa regeneração da floresta original com plantas de menor porte (DURIGON, 2011).

\section{Uso da terra e mapeamentos dos fragmentos florestais}

O mapeamento do uso e ocupação da terra foi feito a partir da imagem de satélite CBERS 2B/HRC, órbita 151 e ponto 125 . As bandas das imagens foram transformadas para o formato "raw" (sem cabeçalhos) de 8 bits por meio do software SPRING 4.3.2, desenvolvido pelo INPE. A imagem CBERS-2B, por não existir metodologia adequada para correção de efeitos atmosféricos devido à falta de calibração confiável do sensor. Os parâmetros utilizados para a correção da banda $1(0,5-0,8 \mu \mathrm{m})$ foram a longitude e latitude do centro da bacia objeto de estudo, o modelo de atmosfera tropical, modelo continental de aerossóis e para visibilidade horizontal de $16 \mathrm{~km}$.

O georreferenciamento da imagem foi realizado no software SPRING por meio da função registro do programa, sendo utilizado o datum horizontal SIRGAS 2000. As imagens foram submetidas

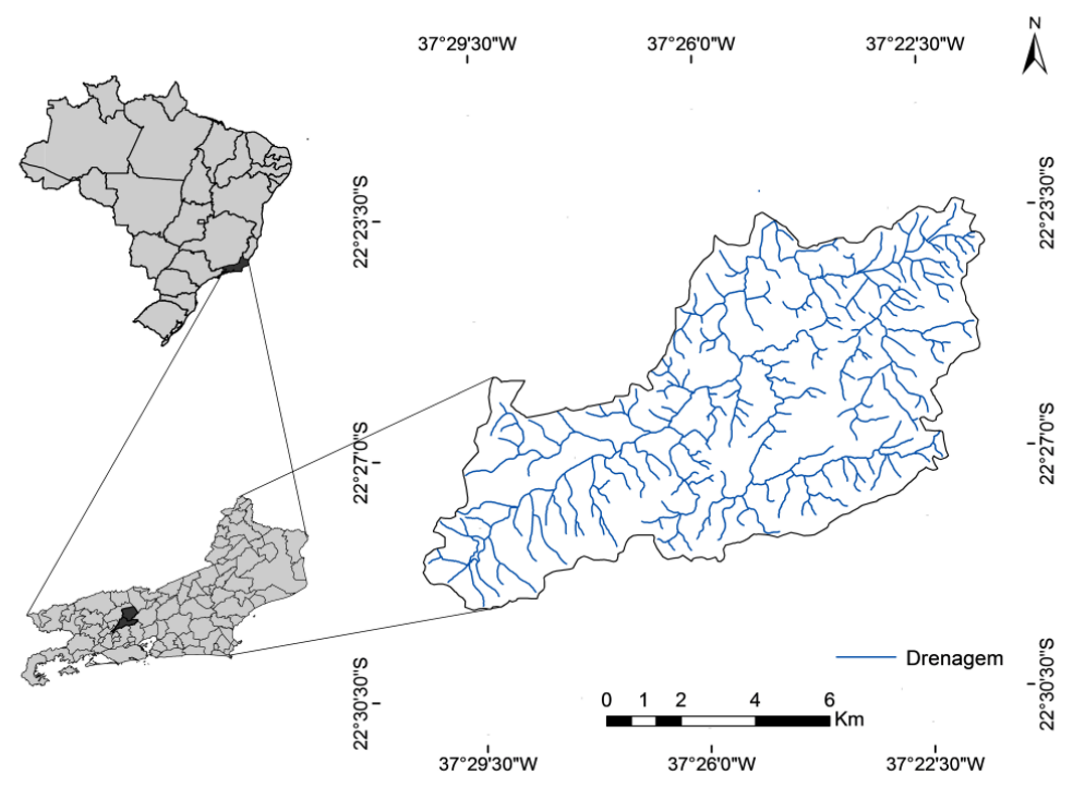

FIGURA 1: Localização geográfica da bacia hidrográfica do Rio Ubá - RJ.

FIGURE 1: Geographical location of the whatershed of Rio Ubá - RJ state. 
à classificação supervisionada por máxima verossimilhança (TSO; MATHER, 2001), na qual foi possível gerar as seguintes classes: capoeira, corpos d'água, floresta secundária, pastagem, solo exposto e urbano.

A partir do mapa de uso e ocupação da terra foi selecionada a classe floresta secundária para serem obtidos os fragmentos florestais presentes, e a partir destes foi quantificada a área de cada fragmento de acordo com a tabela de atributos do próprio arquivo da imagem vetorial, tornando possível comparar os tamanhos dos diversos fragmentos florestais encontrados na Bacia do Rio Ubá. Os fragmentos na área foram relacionados à classe de tamanho a que pertencem: muito pequenos $(<5 \mathrm{ha})$, pequenos $(5-10 \mathrm{ha})$, médios (10-100 ha) e grandes (>100 ha).

\section{Análise dos fragmentos florestais por meio de métricas da paisagem}

A análise dos índices de ecologia da paisagem foi realizada com base no mapa de fragmentos florestais gerado, sendo os valores obtidos pelo software Fragstats ${ }^{\circledR}$ versão 4.2 (MCGARIGAL, 2013). As métricas foram calculadas utilizando a versão para dados em raster para o arquivo de fragmentação florestal obtido na etapa anterior.

Para o cálculo das métricas da paisagem (Tabela 1), foram selecionados índices que possibilitaram quantificar os elementos da paisagem, estimando assim a diversidade, área, densidade, borda, área central, forma, proximidade e isolamento e contágio e dispersão (MCGARIGAL, 2013).

Para melhor comparação entre o grau de conservação e o tamanho dos fragmentos florestais mapeados neste estudo, foi realizada a análise dos índices de ecologia da paisagem para cada uma das classes de tamanho (muito pequeno, pequeno, médio e grande) e para todos os fragmentos, sem distinção de tamanho, a fim de obter valores médios dos índices para todos os remanescentes da área. Por meio das métricas de tamanho, obteve-se a área total da classe fragmento florestal, e o tamanho médio dos fragmentos. Com as métricas de densidade de fragmentos, foi obtido o número de fragmentos em toda a área.

As métricas de forma exigem a adoção de uma paisagem padrão para efeito de comparação. A

TABELA 1: Métricas espaciais utilizadas na quantificação da estrutura da paisagem na Bacia do Rio Ubá - RJ.

TABLE 1: Spatial metrics used to quantify the landscape structure in Ubá whatershed - RJ state.

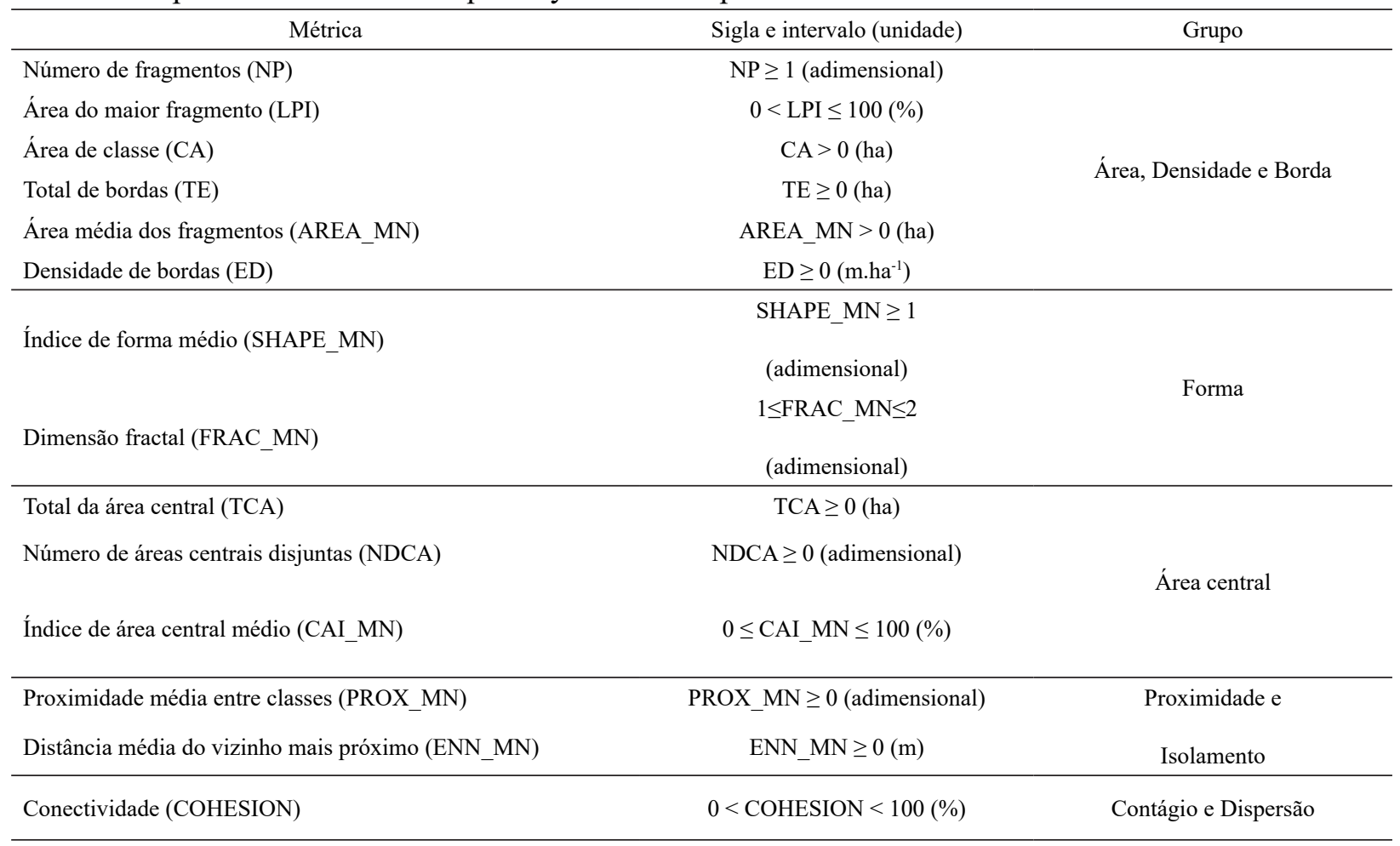


forma dos fragmentos foi comparada com o formato de um círculo para o cálculo da razão perímetro/área, visto que compara o valor desse índice a um círculo quando o arquivo é vetorial e a um quadrado para arquivos raster ou matricial. Os valores de índice de forma mais próximos de 1 são ligados a formatos mais regulares.

Para o cálculo das métricas de área central dos fragmentos, utilizaram-se as distâncias de 20, 40, 60, 80, 100 e 140 m de borda, obtendo diferentes cenários para análises.

\section{RESULTADOS E DISCUSSÃO}

O uso e ocupação da terra da Bacia do Rio Ubá apresentou como maior uso a classe pastagem com $46,28 \%$, as áreas urbanas com $16,81 \%$, corpo d'água com $0,14 \%$ e solo exposto com apenas $0,09 \%$. Quando se somam todos os usos de natureza antrópica, observa-se que $63,32 \%$ da bacia está sob uma matriz antrópica, o que para manutenção dos fragmentos florestais é prejudicial em função de maior efeito de borda (Figura 2).

Em relação ao uso da terra na Bacia do Rio Ubá, a capoeira apresentou 13,03\%, e a floresta secundária com $23,65 \%$ da bacia. A soma do percentual de capoeira e floresta secundária totalizaram $36,68 \%$ da área da bacia, sendo importante estes usos da terra para garantir serviços ambientais, como a manutenção de água nos mananciais, já que a Bacia do Rio Ubá faz parte da Bacia do Rio Paraiba do Sul, principal manancial de água para região metropolitana do estado do Rio de Janeiro.

A delimitação dos fragmentos florestais demonstra que existem 47 fragmentos florestais na Bacia do Rio Ubá (Figura 3). Os fragmentos florestais corresponderam a uma área de 2030,35 ha, aproximadamente $23,65 \%$ de uma área total de 8585,00 ha, podendo ser considerado um bom percentual de cobertura florestal, quando comparado a outras bacias na Mata Atlântica. Por exemplo, quando se compara ao estudo de Pirovani et al. (2014), que observaram que somente $17 \%$ da bacia do Rio Itapemirim - ES, estava recoberta por fragmentos florestais no bioma Mata Atlântica.

Ao se analisarem as classes de tamanho dos fragmentos florestais, os fragmentos muito pequenos e pequenos apresentaram um baixo percentual de 1,0\% e 3,49\%, respectivamente. Os fragmentos médios apresentaram um alto percentual com $76,20 \%$, seguido dos fragmentos grandes que apresentaram $19,31 \%$, o que para a conservação da fauna silvestre e integridade ecológica dos remanescentes florestais é favorável

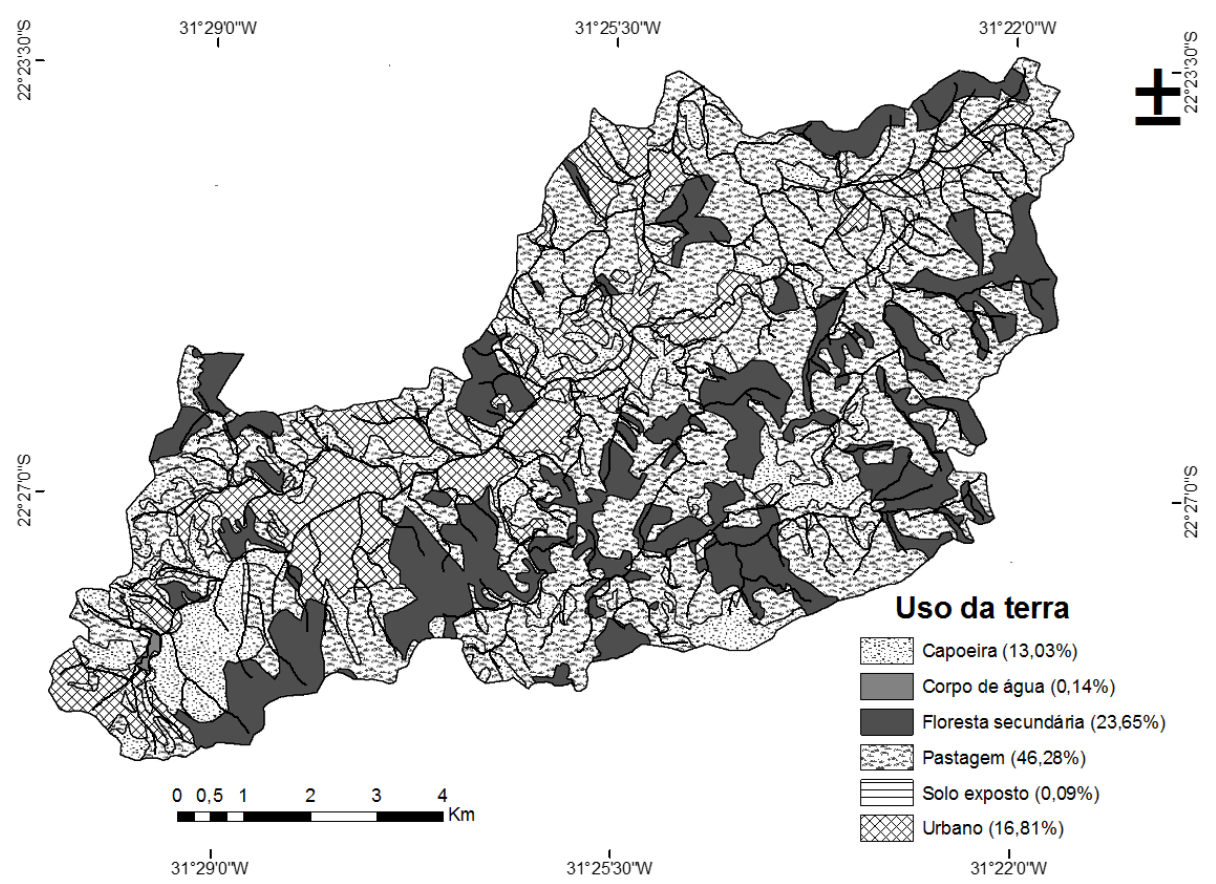

FIGURA 2: Uso e ocupação da terra da Bacia do Rio Ubá - RJ.

FIGURE 2: Land use and cover of Ubá whatershed - RJ state. 


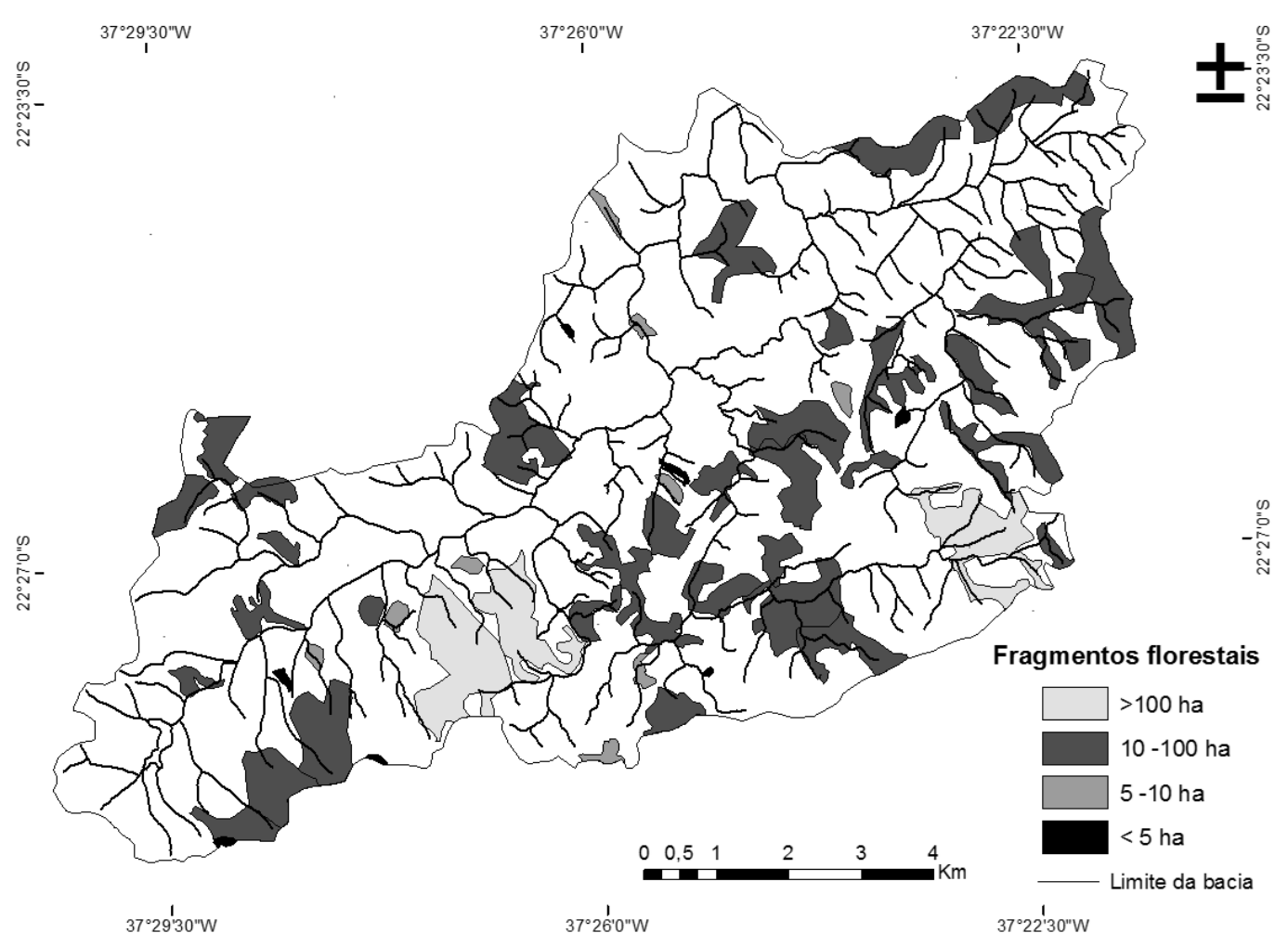

FIGURA 3: Distribuição das classes de tamanho dos fragmentos florestais na área de estudo.

FIGURE 3: Distribution of the size classes of forest fragments in the study area.

(Figura 3). Isto significa que os fragmentos estão bem conservados, já que a riqueza diminui quando a área do fragmento fica menor do que as áreas mínimas necessárias para a sobrevivência das populações.

Os números de fragmentos (NP) de classe de tamanho muito pequeno foram 7 , que corresponderam a $16 \%$ do total, na classe pequeno foram mapeados 9 fragmentos que corresponderam a $21 \%$. A classe de tamanho médio apresentou o maior número com 25 fragmentos em um total de $58 \%$, enquanto a classe de tamanho grande apresentou somente 2 fragmentos, que corresponderam a 5\% (Tabela 2). O número de fragmentos de determinada classe é de fundamental importância para os aspectos dos padrões da paisagem, uma vez que constitui uma medida do seu grau de subdivisão ou fragmentação (CALEGARI et al., 2010).

$\mathrm{Na}$ área de classe (CA), os fragmentos muito pequenos apresentaram uma área de 20,24 ha, os fragmentos pequenos 71,40 ha, os fragmentos médios 1557,10 ha e os fragmentos grandes área de 394,60 ha (Tabela 2). A relação entre o número de fragmentos e a área que eles ocupam é inversa, isto é, os fragmentos da classe de tamanhos pequenos apresentaram maior número de manchas. No entanto, neste estudo observou-se que os fragmentos médios apresentaram maior número de fragmentos (NP) e maior área (CA).

A área do maior fragmento (LPI) na classe muito pequeno foi de $19,71 \%$, na classe pequeno de $13,50 \%$, nos fragmentos médios, a área do maior fragmento foi de $14 \%$ e os grandes fragmentos apresentaram $66,61 \%$, sendo este importante pelo fato de ter um grande fragmento que pode servir como fonte de propágulos e de fauna para os fragmentos menores. Dessa forma, pode-se entender a importância do fragmento-matriz, por ser importante área-fonte de dispersores, polinizadores e propágulos, criando condições favoráveis para a manutenção da biodiversidade local, por meio de processos-chave, como a dispersão, polinização, etc. (CALEGARI et al., 2010).

Os fragmentos grandes apresentaram menor número de fragmentos (NP), o que resulta em uma maior área do fragmento. É notório que a LPI aumenta à medida que o número de fragmentos (NP) diminui o que permite concluir que as áreas dos fragmentos se tornam maiores (SOUZA et al., 2014). 
TABELA 2: Índices de ecologia da paisagem calculados para os fragmentos florestais da Bacia do Rio Ubá - RJ.

TABLE 2: Landscape ecology indexes calculated for the forest fragments of Ubá whatershed - RJ state.

\begin{tabular}{lcccc}
\hline & \multicolumn{4}{c}{ Classes de tamanho } \\
\cline { 2 - 5 } \multicolumn{1}{c}{ Índices } & Muito pequeno & Pequeno & Médio & Grande \\
& $(<5$ ha) & $(5-10$ ha $)$ & $(10-100$ ha $)$ & $(>100$ ha $)$ \\
\hline NP & 16 & 21 & 58 & 5 \\
$0<$ LPI $\leq 100(\%)$ & 19,71 & 13,50 & 14,00 & 66,61 \\
CA $>0$ (ha) & 20,24 & 71,4 & 1557,1 & 394,6 \\
TE $\geq 0$ (ha) & 5678,10 & 12152,52 & 140398,27 & 26757,99 \\
AREA_MN $>0$ (ha) & 2,89 & 7,93 & 62,28 & 197,30 \\
ED $\geq 0$ (m.ha-1) & 281,00 & 169,76 & 90,18 & 67,81 \\
SHAPE_MN $\geq 1(\mathrm{ad})$ & 1,44 & 1,51 & 2,10 & 2,99 \\
FRAC_MN & 1,07 & 1,07 & 1,10 & 1,15 \\
PROX_MN $\geq 0$ (ad) & 0,00 & 0,00 & 128,85 & 0,00 \\
ENN_MN $\geq 0$ (m) & 2069,81 & 1176,97 & 307,25 & 4863,38 \\
$0<$ COHESION $<100(\%)$ & 96,43 & 97,63 & 99,21 & 99,80 \\
\hline
\end{tabular}

Em que: NP (Número de fragmentos); LPI (Área do maior fragmento); CA (Área de classe); TE (Total de bordas); AREA_MN (Área média dos fragmentos); ED (Densidade de bordas); SHAPE_MN (Índice de forma médio); FRAC_MN (Dimensão fractal); PROX_MN (Proximidade média entre classes); ENN_MN (Distância média do vizinho mais próximo); COHESION (Conectividade).

Em relação às métricas de borda, o total de borda (TE) nos fragmentos muito pequenos foi observado o menor valor de TE, enquanto os pequenos e grandes apresentaram TE intermediário, e os fragmentos médios apresentaram maior TE influenciados pela maior área da classe (CA). No entanto, ao comparar o valor de borda com sua contribuição em área dos fragmentos muito pequenos percebe-se que existe maior proporção borda/área que as classes de tamanho maiores. Juvanhol et al. (2011) analisando o total de bordas na Mata Atlântica na região serrana do Espírito Santo, também observaram menor valor de TE nos fragmentos muito pequenos e maior proporção borda/área.

O índice de área média dos fragmentos (AREA_MN) representa a área média dos fragmentos da classe. A AREA_MN no fragmento muito pequeno foi baixa, mas nas demais classes foi observado que a área média dos fragmentos apresentou altos valores, superiores ao limite inferior de cada classe de tamanho. $\mathrm{O}$ fato da maior parte dos fragmentos apresentarem uma área média bem superior ao mínimo de cada classe está ligado à topografia declivosa da região que impede o estabelecimento de pastagens, que é a principal atividade na matriz antrópica que circunda os fragmentos.

Segundo Forman e Godron (1986), os grandes fragmentos são importantes para a manutenção da biodiversidade e de processos ecológicos em larga escala. No entanto, os pequenos fragmentos também cumprem funções relevantes ao longo da paisagem, podendo funcionar como elementos de ligação, trampolins ecológicos (stepping stones) entre grandes áreas, promover aumento no nível de heterogeneidade da matriz e atuar como refúgio para espécies que requerem ambientes particulares que só ocorrem nessas áreas.

A menor classe de tamanho dos fragmentos apresentou a maior densidade de borda (ED) em comparação com as demais classes de tamanho. Observou-se que conforme aumenta a classe de tamanho do fragmento ocorre uma diminuição da densidade de borda. De acordo com Juvanhol et al. (2011), essa diferença da quantidade de bordas, quando se considera a densidade, deve-se aos valores de área ocupados por cada classe de tamanho dos fragmentos, sendo a densidade de bordas inversamente proporcional à área ocupada por cada classe. Esses resultados apontam para um menor efeito de borda nos fragmentos maiores, indicando maior grau de conservação. 
O impacto do efeito de borda está relacionado à forma do fragmento, sendo maior a borda quanto mais irregular o formato, estando este diretamente ligado à relação entre o perímetro e a área; quanto menor essa relação, menor também será a borda e vice-versa, fragmentos de formatos mais regulares têm a razão borda/área minimizada e, portanto, o centro da área está mais distante das bordas. Assim, a área central encontra-se isolada dos fatores externos, enquanto fragmentos de formatos mais irregulares têm maior proporção de bordas, fazendo que ocorra maior interação com a matriz circundante (PIROVANI et al., 2014).

$\mathrm{O}$ índice de forma médio (SHAPE_MN) demonstrou que os fragmentos muito pequenos e pequenos apresentaram formato mais regulares (SHAPE_MN $=1,44$ e 1,51 , respectivamente) quando comparados com os fragmentos médios e grandes, que apresentaram valores de 2,10 e 2,99, respectivamente (Tabela 2). Desta forma, o aumento do tamanho dos fragmentos resulta em formatos mais irregulares. Apesar dos fragmentos menores serem mais suscetíveis ao efeito de borda que os maiores, neste estudo foi observado o contrário, com os maiores fragmentos sofrendo maior efeito de borda em função do formato mais irregular. Pirovani et al. (2014) observaram comportamento semelhante, no qual o índice de forma médio revelou que os fragmentos pequenos possuíam formato mais regular (SHAPE_MN $=1,85$ ) quando comparados com os fragmentos médios e grandes que apresentaram valores de SHAPE_MN de 2,50 e 4,29, respectivamente.

A dimensão fractal média (FRAC_MN) apresentou valores semelhantes nas diferentes classes de tamanho, nas quais os valores observados estão próximos de 1 demonstrando que os fragmentos florestais têm formas pouco irregulares com perímetro simples. Pirovani, Silva e Santos (2015) observaram que a dimensão fractal média na RPPN Cafundó no Espírito Santo de maneira geral, mostraram-se pouco irregulares por apresentarem valores mais próximos de 1 .

A proximidade média entre classes (PROX_MN) nos fragmentos muito pequenos, pequenos e grandes apresentaram valores de zero, um raio de busca de $100 \mathrm{~m}$. Isto significa que, considerando um raio de busca de $100 \mathrm{~m}$, os fragmentos muito pequeno, pequeno e grande não apresentaram proximidade em uma distância de até $100 \mathrm{~m}$, a classe dos médios fragmentos apresentou um valor satisfatório sendo superior a zero.

O nível de isolamento dos fragmentos florestais na paisagem pode ser expresso pela distância média do vizinho mais próximo (ENN_MN), ou seja, quanto maior o valor de ENN_MN maior será o isolamento (MCGARIGAL, 2013). Na distância média do vizinho mais próximo (ENN_MN), os fragmentos médios apresentaram a menor distância, ao passo que os fragmentos grandes, pequenos e muito pequenos apresentaram as maiores distâncias. Os fragmentos de tamanho médios possuem um baixo grau de isolamento, devido ao menor ENM_MN dos fragmentos, sendo a única classe que apresentou uma proximidade (PROX_MN) menor que $100 \mathrm{~m}$ entre fragmentos, embora estes estejam inseridos em uma matriz antrópica constituída principalmente por pastagem, com baixa permeabilidade entre usos da terra e os fragmentos florestais dificultando a conectividade da fauna e flora.

A conectividade foi calculada utilizando o índice COHESION, que calcula a conectividade física dos fragmentos. A conectividade (COHESION) em todas as classes de tamanho dos fragmentos florestais apresentou uma grande conectividade, sendo favorável para a fauna e flora mantendo o fluxo entre os fragmentos florestais. Souza et al. (2014) observaram altos valores de COHESION na Área de Proteção Ambiental Coqueiral em Minas Gerais, com um percentual de 97,32\%, valor próximo ao observado neste estudo, demonstrando que apesar da paisagem fragmentada, os fragmentos estão ligados por estruturas físicas como corredores.

As métricas relativas às áreas centrais (núcleo) dos fragmentos estão expressas na Tabela 3, objetivando investigar qual faixa de borda exerce maior influência na área central dos fragmentos.

O TCA mede a quantidade de área central da classe considerada, e as espécies que ocupam a área central (interior do fragmento) não estão sujeitas aos mesmos impactos que aquelas inseridas na borda do fragmento sobre influência da matriz antrópica (MCGARIGAL, 2013). Em relação ao total da área central (TCA), observou-se que conforme aumenta o efeito de borda reduz-se o total de área central, sendo igual a zero nos fragmentos muito pequeno e pequeno para as maiores distâncias de borda. Os fragmentos da classe médio e grande em função de sua maior área, mesmo nas maiores distâncias de borda, mantiveram sua área central. 
TABELA 3: Índices de ecologia da paisagem relativos às métricas de área central.

TABLE 3: Landscape ecology indices relating to the metrics of the central area.

\begin{tabular}{|c|c|c|c|c|c|c|}
\hline \multirow[t]{2}{*}{ Distância de borda (m) } & \multicolumn{3}{|c|}{ Muito pequeno $(<5 \mathrm{ha})$} & \multicolumn{2}{|c|}{ Pequeno (5-10 ha) } & \multirow[b]{2}{*}{ CAI_MN } \\
\hline & TCA & NDCA & CAI_MN & TCA & NDCA & \\
\hline 20 & 11,32 & 7 & 54,26 & 51,22 & 9 & 71,85 \\
\hline 40 & 3,97 & 7 & 17,57 & 31,92 & 10 & 44,84 \\
\hline 80 & 0,07 & 1 & 0,26 & 6,70 & 9 & 9,21 \\
\hline 100 & 0,00 & 0 & 0,00 & 1,64 & 7 & 2,14 \\
\hline 140 & 0,00 & 0 & 0,00 & 0,00 & 0 & 0,0 \\
\hline \multirow[t]{2}{*}{ Distância de borda (m) } & \multicolumn{3}{|c|}{ Médio (10-100 ha) } & \multicolumn{3}{|c|}{ Grande (>100 ha) } \\
\hline & TCA & NDCA & CAI_MN & TCA & NDCA & CAI_MN \\
\hline 20 & 1325,92 & 27 & 78,63 & 347,96 & 2 & 87,23 \\
\hline 40 & 1089,14 & 43 & 61,09 & 298,85 & 5 & 73,86 \\
\hline 80 & 679,36 & 52 & 32,75 & 215,88 & 8 & 51,67 \\
\hline 100 & 518,33 & 52 & 22,52 & 184,38 & 6 & 43,64 \\
\hline 140 & 269,53 & 39 & 10,72 & 136,73 & 3 & 32,11 \\
\hline
\end{tabular}

Em que: TCA (Total da área central); NDCA (Número de áreas centrais disjuntas); CAI_MN (Índice de área central médio).

Com a fragmentação excessiva, ocorre aumento da área da borda em relação à área do fragmento. Por isso, a relação perímetro/área aumenta e, se o fragmento continua a diminuir de tamanho, pode acabar se tornando inteiramente um habitat de borda (JUVANHOL et al., 2011).

A métrica número de áreas centrais disjuntas (NDCA) apresentou uma redução conforme aumentou a distância da borda nos fragmentos muito pequeno e pequeno, nos fragmentos médio e grande apresentaram uma redução, porém, não se aproximando do valor zero. Pirovani et al. (2014) avaliando diferentes distâncias de bordas observaram o decréscimo do número de áreas centrais disjuntas com o aumento da distância de borda, no qual a partir dos $100 \mathrm{~m}$ houve uma redução significativa do número de áreas centrais disjuntas dos fragmentos.

O índice de área central médio (CAI_MN) apresentou um padrão semelhante às métricas TCA e NDCA, com redução dos valores conforme se aumentou a distância de borda. Os fragmentos muito pequenos e pequenos apresentaram valores de zero para as maiores distâncias de borda. Em relação ao tamanho do fragmento, observou-se que os fragmentos de menor tamanho (muito pequeno e pequeno), quando se utilizam as maiores distâncias de borda apresentaram valores nulos.

$\mathrm{Na}$ distância de borda de $80 \mathrm{~m}$, os fragmentos muito pequeno e pequeno apresentaram uma redução drástica das métricas de área central, diminuindo o valor do índice de área central total a 0,07 e 6,70 ha, respectivamente. Os fragmentos, independentemente de seu tamanho, apresentaram uma redução do CAI_MN, conforme se aumenta a distância de borda. Observa-se que os fragmentos médios apresentaram maior valor para NDCA, o que está de acordo com maior NP.

Nas classes de tamanho dos fragmentos florestais, o aumento da distância de borda de $20 \mathrm{~m}$ para 40 m não representa nenhuma alteração nos valores das métricas de área central, ou seja, o acréscimo de 20 $m$ na distância de borda não altera as formas dos fragmentos, mantendo o mesmo valor de área central. A simulação com a distância de borda de $100 \mathrm{~m}$ gerou redução mais drástica nas métricas de área central dos fragmentos muito pequenos e pequenos, e os fragmentos médios e grandes sofreram menores reduções de área central para essa distância de borda, apresentando TCA de 518,33 e 184,38 ha, respectivamente.

A distância de borda de $140 \mathrm{~m}$ levou os índices de área central dos fragmentos muito pequenos e pequenos a resultados nulos, sendo o TCA 0,00 ha, respectivamente para ambas as classes de tamanho. Portanto, os fragmentos muito pequenos e pequenos estão totalmente sob efeito de borda, estando os fragmentos suscetíveis à influência da matriz antrópica. 
Na classe de tamanho de fragmentos médios e grandes, o aumento na distância de borda apresentou redução nas métricas de áreas centrais de menor magnitude e de forma mais gradual. O índice de área central total dos fragmentos médios foi de 518,33 ha e 269,53 ha, considerando uma distância de borda de $100 \mathrm{~m}$ e $140 \mathrm{~m}$, respectivamente. Nos fragmentos grandes, quando utilizada uma distância de borda de 100 m e 140 m, o índice de área central total foi de 184,38 ha e 136,73 ha, respectivamente.

Observa-se nas classes de tamanho médios e grandes que a simulação com a maior distância de borda (140 m) não acarreta a um parcial ou total efeito de borda nos fragmentos, em contraste ao observado nos fragmentos pequenos e muito pequenos. Isto ocorre nos fragmentos médios e grandes pelo aumento do CAI_MN para estas classes de tamanho.

\section{CONCLUSÃO}

A Bacia do Rio Ubá possui o predomínio de uma matriz antrópica por pastagem, pequeno percentual de cobertura florestal com fragmentos de tamanho médio, com alto grau de fragmentação florestal da paisagem.

Os fragmentos médios apresentaram-se como a classe mais numerosa e com maior área total, e a única classe de tamanho que mantém uma distância menor que $100 \mathrm{~m}$ entre si. Os fragmentos muito pequenos e pequenos têm formatos mais regulares, porém, com maior densidade de borda.

Em relação às métricas de área central, os fragmentos muito pequeno e pequeno perderam totalmente sua área central, estando completamente sob efeito de borda considerando uma distância maior.

Uma alternativa no contexto da Bacia do Rio Ubá é a utilização dos fragmentos florestais muito pequenos e pequenos como corredores ecológicos para interligação aos fragmentos médios.

\section{REFERÊNCIAS}

BEZERRA, C. G. et al. Estudo da Fragmentação Florestal e Ecologia da Paisagem na sub-bacia hidrográfica do Córrego Horizonte, Alegre, ES. Espaço e Geografia (UnB), Brasília, v. 14, n. 2, p. 257-277, 2011.

CALEGARI, L. C. et al. Análise da dinâmica de fragmentos florestais no município de Carandaí - MG, para fins de restauração florestal. Revista Árvore, Viçosa, MG, v. 34, n. 5, p. 871-880, 2010.

FORMAN, R. T. T.; GODRON, M. Landscape ecology. Washington: John Wiley, 1986.120 p.

DEVIDE, A. C. P. et al. História ambiental do Vale do Paraíba Paulista, Brasil. Revista Biociências, Porto Alegre, v. 20, n. 1, p. 12-29, 2014.

DURIGON, V. L. Utilização do geoprocessamento e do sensoriamento remoto na determinação da susceptibilidade do solo à erosão em bacias hidrográficas, associada ao índice de vegetação. 2011. 134 f. Tese (Doutorado em Agronomia - Ciências do Solo) - Universidade Federal Rural do Rio de Janeiro, Seropédica, 2011.

ETTO, T. L. et al. Ecologia da paisagem de remanescentes florestais na Bacia Hidrográfica do Ribeirão das Pedras - Campinas-SP. Revista Árvore, Viçosa, MG, v. 37, n. 6, p. 1063-1071, 2013.

JUVANHOL, R. S. et al. Análise Espacial de Fragmentos Florestais: caso dos Parques Estaduais de Forno Grande e Pedra Azul, estado do Espírito Santo. Floresta e Ambiente, Seropédica, v. 18, n. 4, p. 353-364, 2011.

MCGARIGAL, K. Fragstats: Fragstats help - versão 4.2. Computer software program produced by the author at the University of Massachusetts. Massachusetts: Amherst, 2013. 182 p.

METZGER, J. P. Conservation issues in the Brazilian Atlantic Forest. Biological Conservation, Essex, v. 142, n. 6, p. 1138-1140, 2009.

PIROVANI, D. B. et al. Análise espacial de fragmentos florestais na Bacia do rio Itapemirim, ES. Revista Árvore, Viçosa, MG, v. 38, n. 2, p. 271-281, 2014.

PIROVANI, D. B.; SILVA, A. G.; SANTOS, A. R. Análise da paisagem e mudanças no uso da terra no entorno da RPPN Cafundó, ES. Cerne, Lavras, v. 21, n. 1, p. 27-35, 2015.

RIBEIRO, M. C. et al. The Brazilian Atlantic Forest: a shrinking biodiversity hotspot. In: ZACHOS, F. E.; HABEL, J. C. (Ed.). Biodiversity hotspots: distribution and protection of conservation priority areas. Heidelberg: Springer, 2011. p. 234-243. 
RIBEIRO, M. C. et al. The Brazilian Atlantic Forest: how much is left, and how is the remaining forest distribuited? implications for conservation. Biological Conservation, Essex, v. 142, n. 6, p. 1141-1153, 2009.

SILVA, L. G.; SANTOS, S.; MORAES, F. Fragmentação da Mata Atlântica de interior: análise de paisagem do corredor verde Sul-Americano e Florestas do Alto Paraná. Boletim geográfico. Maringá, v. 32, n. 3, p. 61-68, 2014.

SILVA, M. S. F.; SOUZA, R. M. Padrões espaciais de fragmentação florestal na FLONA do Ibura - Sergipe. Mercator, Fortaleza, v. 13, n. 3, p. 121-137, 2014.

SOARES, V. P. et al. Mapeamento das áreas de preservação permanente e dos fragmentos florestais naturais como subsidio à averbação de reserva legal em imóveis rurais. Cerne, Lavras, v. 17, n. 4, p. 555-561, 2011. SOUZA, G. C. et al. Análise da fragmentação florestal da Área de Proteção Ambiental Coqueiral, Coqueiral - MG. Ciência Florestal, Santa Maria, v. 24, n. 3, p. 631-644, 2014.

TABARELLI, M. et al. Prospects for biodiversity conservation in the Atlantic Forest: lessons from aging human-modified landscapes. Biological Conservation, Essex, v. 143, n. 10, p. 2328-2340, 2010.

TSO, B.; MATHER, P. M. Classification methods for remotely sensed data. New York: Taylor \& Francis, 2001. $332 \mathrm{p}$. 